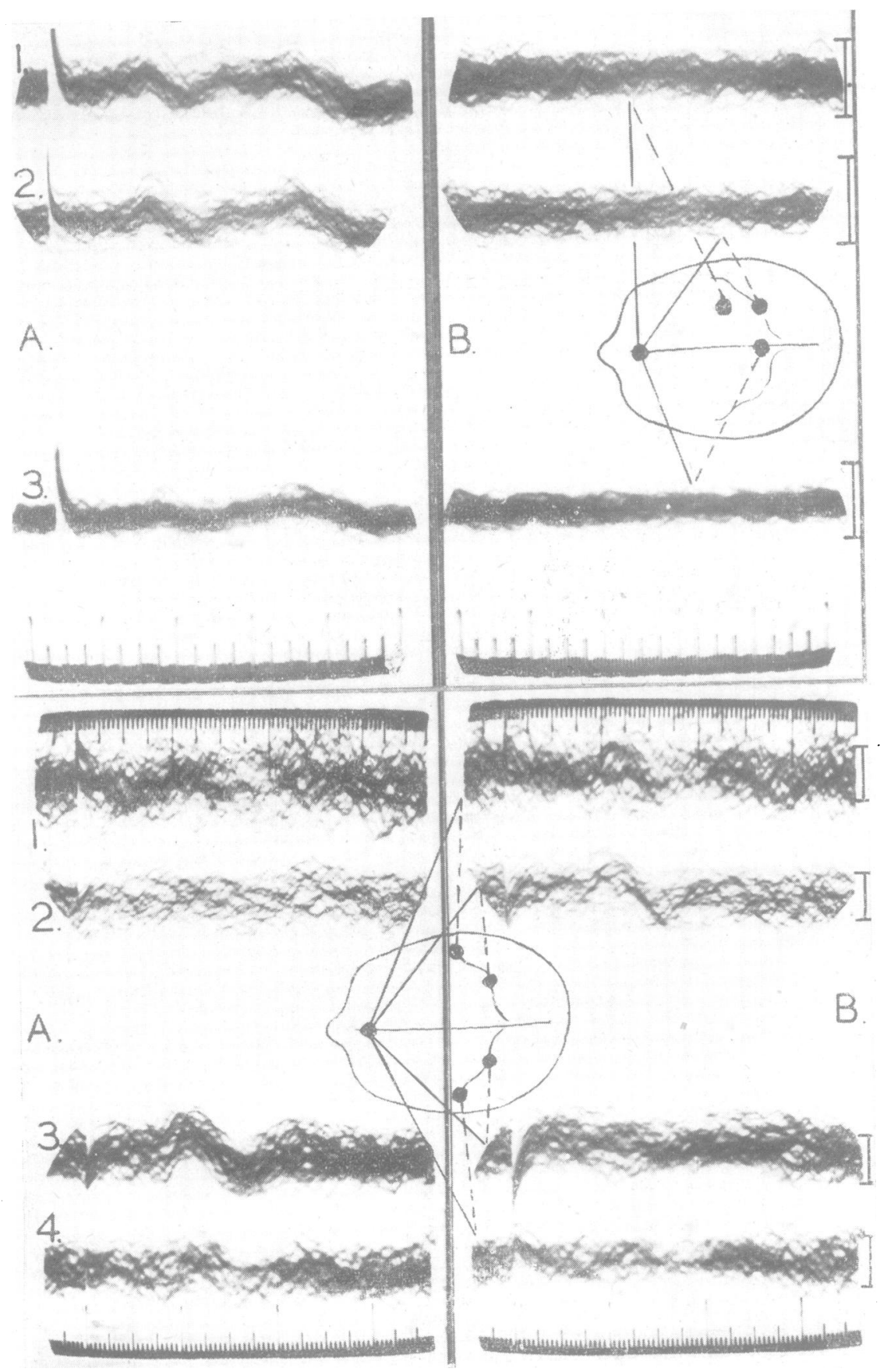

Fig. 1.-In (a) are shown, superimposed, fifty responses to stimulation of the left ulnar nerve at the wrist. The stimulus 0 escape at the left-hand end of the record is followed by a train of $\overline{\bar{c}}$ waves each with a period of about 20 msecs. In $\leqslant$ (b) the potential differ- $\Rightarrow$ ences between the same $s$ electrodes on the head 0 have been recorded but $\frac{\bar{\sigma}}{\sigma}$ no stimulus has been applied. No deflections comparable with those $\mathbb{Q}$ in (a) occur. The time scales show intervals of os 5 and 20 msecs. Cali- $\overrightarrow{0}$ bration marks at the right-hand end of (b) $\vec{\omega}$ show the deflection pro- $\sigma$ duced in each channel by a potential difference of $20 \mu \mathrm{V}$.

Fig. 3.-The records shơw the responses to stimu每 tion of the ulnar nerkes at the elbow. In $(a)$ the stimulus is on the riste side and the only visibe responses appear in leads 3 and 4 from the left hemisphere. In $(b)$ thes stimulus is applied to the left side and the response is only apparento in leads 1 and 2 from the right side of the head $\overrightarrow{ }$ The posterior electrodeso are 7 and $14 \mathrm{~cm}$. from $?$ the midline and in each case the response iso largest in the lead from the electrode $7 \mathrm{~cm}$. from the midline. The calio bration lines at the end of (b) represent $20 \mu \mathrm{V} 3$ 


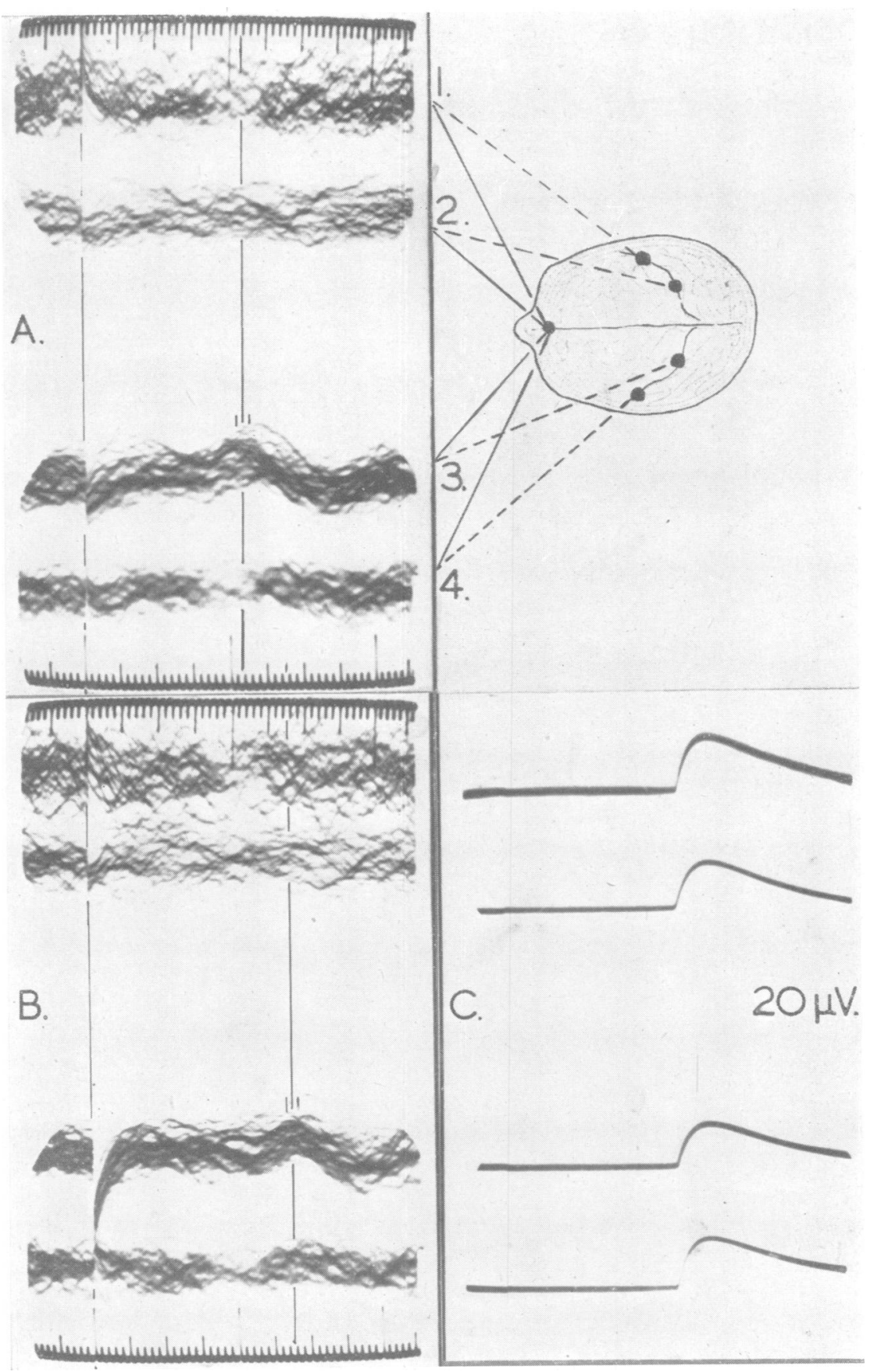

Fig. 2.-The records show the responses to stimulation of the right ulnar nerve. In (a) the stimulus has been applied at the elbow and the latency from the stimulus to the peak of the response is $22 \pm 1$ msecs. In $(b)$ the stimulus has been applied at the wrist and the latency is $28 \pm 1$ msecs. (c) shows a record of fifty superimposed deflections due to a rectangular step input to the amplifiers of $20 \mu \mathrm{V}$. This record indicates the amplifier frequency response used in these experiments and the small contribution of the amplifier irregularity to the thickness of the baseline in the records shown in $(a)$ and $(b)$. The time scales in this and all following records show 1,5 , and $20 \mathrm{msec}$. intervals. 


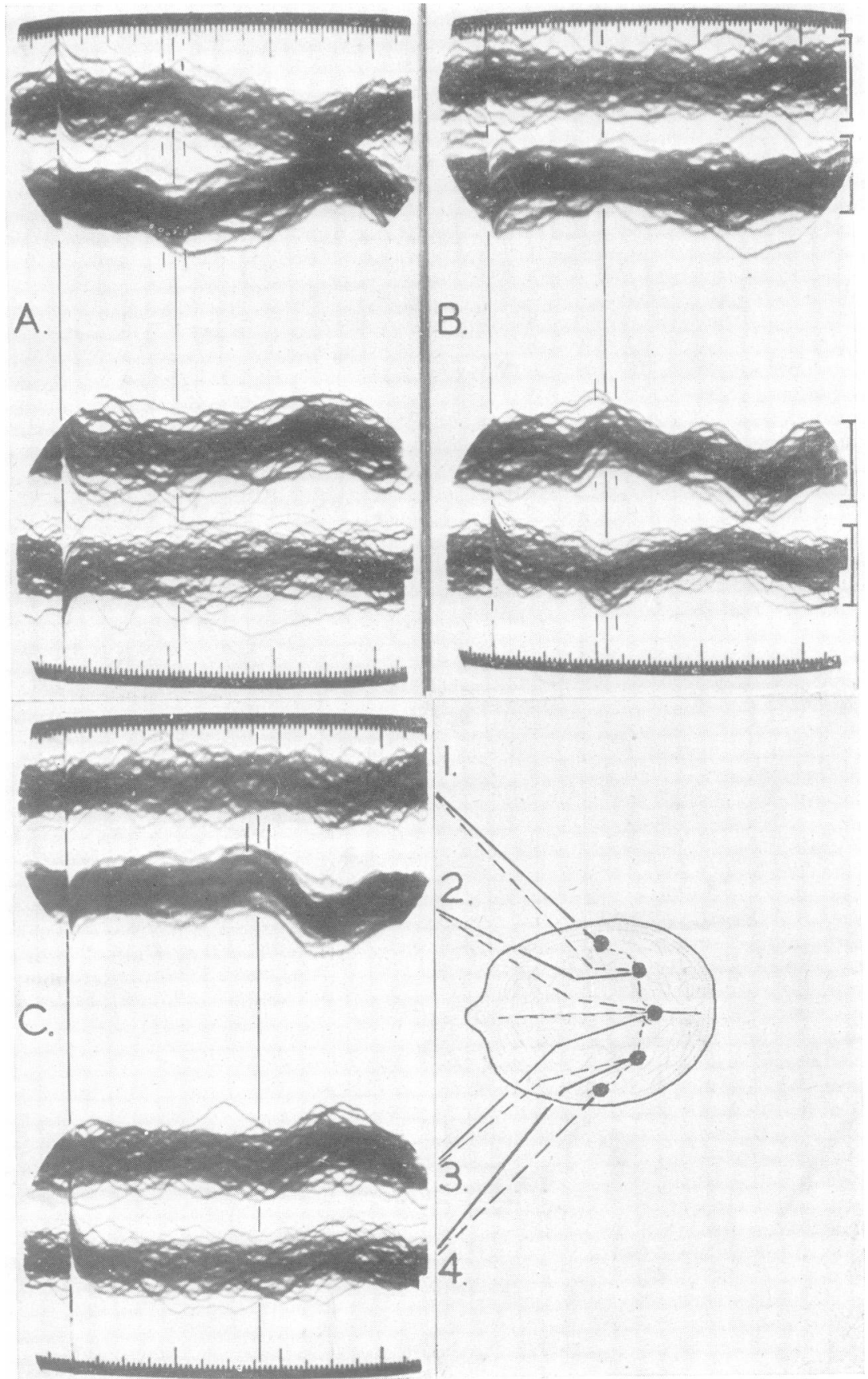

FIG. 4.-The records show the responses to stimulation of the left and right median nerves at the elbow and the left lateral popliteal nerve at the head of the fibula. The electrodes are applied over the surface markings of the sensorymotor areas in the midline and 6 and $12 \mathrm{~cm}$. from it on each side. A stimulus to the left median nerve (a) produces a potential change maximum near to the electrode on the right side common to leads $t_{0} \omega$ and $2,6 \mathrm{~cm}$. from the $\vec{\omega}$ midline. A stimulus to the right median nerve 0 (b) produces a simila甲 disturbance on the leff side of the head. Stimuk lation of the left lateras popliteal nerve gives rise $\frac{5}{\omega}$ to a potential change. occurring nearer to the $\overrightarrow{0}$ midline electrode than t any of the others, but not symmetrically disposed with respect to it. The latency of the response to stimulation in the leg is $36 \pm 2$ msecs., about 14 msecs. longer than in (a) and $(b)$. The calibration marks show a $\overline{\hat{0}}$ potential difference of 3 $15 \mu \mathrm{V}$. 


\title{
CEREBRAL RESPONSES TO ELECTRICAL STIMULATION OF PERIPHERAL NERVE IN MAN \\ BY
}

\author{
G. D. DAWSON
}

The Neurological Research Unit of the Medical Research Council, National Hospital, Queen Square

\author{
(RECEIVED 30TH JuLy, 1947)
}

\section{Introduction}

EXPERiMENTS to be described elsewhere have shown that in a patient suffering from myoclonic seizures electrical stimulation of peripheral nerve resulted in changes of electrical potential detectable on the scalp. These changes in potential difference were largest over the hemisphere on the side opposite to that stimulated. They were located near to the midline when the lateral popliteal nerve in the leg was stimulated and more laterally when the ulnar nerve was stimulated in the arm.

The experiments to be described in this paper have been made to find out if any action potentials may be detected in 'healthy subjects comparable with those found in the patient with myoclonus. Evidence will be presented to support the suggestion that potential changes of cerebral origin, which probably arise in the central or postcentral cortex, may be detected on the scalp in healthy subjects following electrical stimulation of peripheral nerves.

\section{Methods}

The stimulus used was an electric shock with a rapid rise and a decay of exponential form with a time constant of 50 microseconds. The cathode, a $1 \mathrm{~cm}$. diameter pad soaked in salt solution and mounted on a chloridecoated silver rod, was applied to the ulnar nerve at the wrist or elbow, to the median nerve at the elbow, or to the lateral popliteal nerve at the head of the fibula. The anode was a saline-soaked pad large enough to cover the back of the hand and applied either there, to the forearm, or to the calf of the leg. Neither of the stimulating electrodes was earthed, and the stimulus was applied to them through a Muirhead wide-range transformer. An earth connexion was made the to subject, usually to the upper part of the chest or on the limb stimulated proximal to the stimulating electrodes. The stimulus was adjusted in size to be maximal or supramaximal for the motor fibres in the nerves stimulated. It was applied once a second by a trigger circuit locked to a fixed point on the sweep of a cathode ray oscillograph. Fifty or more successive sweeps were photographed superimposed in a single record. In such a record any waves which follow the stimulus by a regular interval will all occur at the same place on the trace ; they may thus be detected although they are no larger than the deflections produced by spontaneous activity of the brain and scalp muscle. As deflections due to these causes are not regularly related to the stimulus, they tend to occur at a different place on each successive sweep and therefore appear only as a thickening of the baseline. High contrast obtained in the photographic processing makes clearer the places where a number of sweeps have superimposed exactly. The time scales on the records show 5 and 20 millisecond intervals in Fig. 1 and in all other figures intervals of 1,5 , and 20 msecs. In the earlier experiments these time scales were derived from the $50 \mathrm{c} / \mathrm{s}$. supply mains but in later experiments, for reasons connected with the exclusion of artefacts which will be discussed later, they were derived from a quartz crystal oscillator. The sizes of the calibration marks on the records are indicated below each figure. All records read from left to right.

At first the scalp potentials were picked up with saline-pad electrodes. For reasons of comfort in the later experiments, which tended to be longer, silver-cup electrodes were fixed on with collodion. The cups were $1 \mathrm{~cm}$. in diameter, coated with silver chloride and filled with Cambridge electrode jelly. The electrode placements used are indicated in the figures. The amplifiers were connected in such a way that, if in any channel the electrode shown connected to the continuous line became negative with respect to that shown connected to the broken line in the head diagrams, an upward deflection would result in the record from that channel. The potential differences were amplified with conventional resistance-capacity coupled amplifiers and recorded photographically from Cossor double-beam cathode-ray oscillographs. The frequency response of the amplifiers was restricted so that they attenuated, with respect to the level at $35 \mathrm{c} / \mathrm{s}$., by 50 per cent. at $180 \mathrm{c} / \mathrm{s}$. and by 20 per cent. at $10 \mathrm{c} / \mathrm{s}$. This restriction of the frequency response must have affected the form of some of the waves recorded. Therefore little importance has been attached to waveform in these studies, though experiments with less restricted frequency responses suggest that the distortion is not gross. No resonant circuits have been used to restrict the frequency response, and therefore any periodic waves in the records are not due to the excitation of such circuits by spread from the stimulus or any other regularly occurring disturbance.

The subjects were examined in the supine position 
with the head supported in such a way as to relax the neck muscles. During the periods of stimulation the eyes were kept open or closed, whichever reduced to the greatest extent the combination of spontaneous brain activity, scalp muscle activity, and blinking, all of which thicken the baseline.

\section{Material}

Fourtèen subjects have been examined. All were healthy males between the ages of 26 and 50 years. No drugs were given and no abnormal conditions were present at the time of the examinations.

\section{Results}

In twelve of the fourteen subjects examined it has been possible to detect a change of potential over the surface marking of the contralateral sensory area following stimulation of peripheral nerves.

Fig. 1A (p. 134) is a photograph of the superimposed responses to fifty successive stimulations, at one second intervals, of the left ulnar nerve at the wrist. The record shows a series of small but definite waves following the initial rapid deflection due to spread from the stimulus. The first clearly discernible wave begins 22 to 23 msecs. after the stimulus and the first peak occurs 25 to 27 msecs. after the stimulation. The sense of the deflection of the first phase of the waves indicates that the more posterior electrodes became positive with respect to the common electrode at the nasion. The deflections are greatest in leads 1 and 2 , from the electrodes lateral to the midline, and least in lead 3 , from the electrode on the midline. This difference in the deflections in the different leads suggests that the disturbance did not occur under the frontal electrode common to all three channels. Fig. 1B shows fifty successive sweeps recorded under the same conditions as in Fig. 1A but without any stimulus applied to the nerve. No comparable deflections occur, and it may therefore be suggested that the waves following the stimulus in Fig. 1A were provoked by that stimulus and were not spontaneous. Before it can be decided that such waves are of cortical origin several sources of artefact must be excluded.

The period of the waves in Fig. 1A is near to $1 / 50$ th of a second, and it is therefore necessary to show that they are not due to any form of pickup from the $50 \mathrm{c} / \mathrm{s}$. supply mains. In Fig. 1B, where no stimulus was applied, there is some evidence towards the end of the trace of a small oscillation with a frequency of $100 \mathrm{c} / \mathrm{s}$., which may well have had such an origin. The time scale in these first records was derived from the $50 \mathrm{c} / \mathrm{s}$. supply mains, and to obtain superimposition of the time marks in the successive sweeps the recurrence rate of the sweep had necessarily to be an exact sub-multiple of the mains frequency. Therefore waves related integrally to the supply frequency superimposed accurately. To avoid this difficulty $\stackrel{\mathbb{D}}{\frac{1}{2}}$ due to interference from the mains the sweep was in all later experiments locked to a sub-multiple of $50.00 \mathrm{c} / \mathrm{s}$. derived from a quartz crystal oscillator which also provided the time scale. It is usuallyo possible to record at a time when the frequency of the mains is not exactly $50 \mathrm{c} / \mathrm{s}$. At such a time no two waves due to potentials derived from the mains will superimpose exactly, except at long intervals, $\stackrel{\frac{\rho}{\hat{S}}}{\text {. }}$ and consequently they will appear as a thickening of the baseline and not as a regular wave. Thus any. waves which superimpose accurately in successive sweeps cannot be derived from the mains if thesehave a frequency of other than $50 \mathrm{c} / \mathrm{s}$. at the time the $\frac{C}{0}$ record is made. Using this method it is possible $\frac{\bar{\sigma}}{\omega}$ to say that the waves following the stimulations in $\overrightarrow{\mathbb{C}}$ the later records were not due to any form of interference from the supply mains.

The next source of artefact that must be excluded $\stackrel{\circ}{\circ}$ is spread of the stimulating current. The potential $\vec{\omega}$ difference between the stimulating electrodes in 2 these experiments was between 50 and 80 volts ; and it is not always possible to avoid all spread of current ${ }^{\text {? }}$ to the recording electrodes. That the train of waves $\vec{\circ}$ following stimulation is not just a residual oscillation $\omega$ due to spread of the stimulus is suggested by the records shown in Figs. 2 and 4 (pp. 135, 136). Theo records in Fig. 2 were made from four electrodes two over each sensory-motor region, and a fifth of common to all four channels at the nasion. Stimtr-c lation of the right ulnar nerve at the elbow, Fig. 2 엉 produced a response visible in lead 3 , from the more? medial of the two electrodes on the left side of the head. The interval between the stimulus and the. peak of the first phase of the wave, indicated by the vertical lines through all four traces, is $22 \pm 1$ msecs. The short vertical lines over the peak of the wave indicate intervals of $1 \mathrm{msec}$. before and after the long line. In this and all later figures the stimulus $\stackrel{\mathbb{Q}}{\unrhd}$ was released at the time of the first 20 msec. marker $\overrightarrow{\vec{B}}$ in the time scale. Stimulation of the same nerve $\frac{0}{3}$ but at the wrist, Fig. 2B, produced a slightly smaller response in the same lead; and the interval between $\overrightarrow{0}$ the stimulus and the first peak of the wave is now $28 \pm 1$ msecs. This increase in the latency of the response by $6 \pm 2$ msecs. suggests that the response picked up on the scalp was more probably a correlate of a disturbance propagated in the nerve than of any purely electrical aftermath of the stimulus. The same is suggested by the records in Fig. 4A and C. In Fig. $4 \mathrm{~A}$ a stimulus applied to the left median nerve at the elbow caused a response to appear in leads 1 and $2, \frac{7}{O}$ from the right parietal region. The interval between the stimulus and the first positive peak of the wave is $\mathrm{N}$ $22 \pm 2$ msecs. A stimulus to the lateral popliteal nerve 
at the head of the fibula, Fig. 4c, was followed by a response appearing nearer to the midline, chiefly in lead 2 and to a less extent in lead 3. The interval between the stimulus and the first positive peak of the wave is $36 \pm 2$ msecs., an increase of the latency by $14 \pm 4$ msecs. over that of the response to stimulation of the median nerve at the elbow.

Another source of artefact, a reflex twitch of scalp muscle, cannot be excluded directly, but the locations of the responses to be described make it seem unlikely that this is their origin. For the location of cerebral action potentials through the intact skull the use of a single electrode common to all the amplifiers and as remote as possible from the active area, together with a number of electrodes near to the active area, is open to criticism. This method has, however, the advantage of picking up the largest possible potential differences and it has been used in the majority of these experiments. A more satisfactory method is that described by Adrian and Yamagiwa (1935), who plotted the potential gradients over the scalp. For this a number of electrodes in line are connected to the amplifiers in such a way that a disturbance under an electrode common to two channels produces a deflection in opposite sense in those two channels. Under these conditions, although the place at which the greatest potential change is occurring may be accurately located, the separation of the electrodes is smaller and the potential differences between them may be too small to be detected. Fig. 3 (p. 134) shows records made by the first method with the same electrode positions as those used in the records in Fig. 2. Stimulation of the right ulnar nerve at the elbow, Fig. 3A, produced a response that is largest in lead 3, from the more medial of the two electrodes on the left side of the head, $7 \mathrm{~cm}$. from the midline. No response is evident in leads 1 and 2 from the head on the same side as the arm stimulated. Transferring the stimulus to the ulnar nerve of the left side, Fig. 3B, caused the response to appear largest in lead 2, from the more medial of the two electrodes on the right side of the head. No response was then evident in leads 3 and 4, from the left side of the head. It seems that in this experiment the response was largest about $7 \mathrm{~cm}$. from the midline on the side of the head opposite to that of the arm stimulated. The second method of location was used in the experiment from which records are shown in Fig. 4. Here a line of electrodes was applied over the surface markings of the sensory-motor -areas of the two sides. The lateral electrodes were 6 and $12 \mathrm{~cm}$. from the midline. The record in Fig. 4A shows a deflection occurring in opposite sense in channels 1 and 2, from the right side of the head, when the left median nerve was stimulated at the elbow. This shows that the first phase of the response, with its peak 22 msecs. after the stimulus, was a disturbance that was greatest nearer to the electrode $6 \mathrm{~cm}$. from the midline on the right side than to any of the other electrodes; also that in this phase of the response the active area became positive with respect to the parts of the scalp under the other electrodes. Stimulating the median nerve at the elbow on the right side, Fig. 4B produced a similar response but maximal about $6 \mathrm{~cm}$. from the midline on the left side of the head. Fig. $4 \mathrm{C}$ shows records taken during stimulation of the lateral popliteal nerve at the head of the left fibula. Here the response is largest in lead 2 and of opposite sense, though much smaller in lead 3. This indicates that the disturbance occurred nearer to the midline electrode than to any of the others but was not symmetrically disposed with respect to this electrode.

In none of these healthy subjects who showed the response was it possible to pick up the potential changes more than two or three centimetres in front of or behind the surface markings of the central sulcus. The locations of the responses to stimulation of peripheral nerves, particularly that in which the response to stimulation of the popliteal nerve in the leg was located near the midline, where there is least scalp muscle, seem to indicate that the responses were probably not due to reflex twitches of scalp muscle.

Experiments on the subject. with myoclonic seizures, to be described elsewhere, have shown that the response to a stimulus can be facilitated to the extent of 50 to 70 per cent. in size by the previous application of a conditioning shock 60 to 100 msecs. earlier. The period of facilitation is followed by a period in which the response to the test shock is depressed. Experiments were made with paired shocks in four of the healthy subjects in this series. So far no objective evidence of any facilitation of the response to the test shock has been found. However, each of the four subjects, although unaware of the results obtained with the others, or of the manner in which the interval between the stimuli was changed, described an alteration in the quality of the perceived sensation when the interval between the stimuli was between 70 and 100 msecs. The first subject stated that there was something wrong with the stimulator, since he was feeling four instead of two shocks, which he had reported at longer and shorter intervals. The other three subjects described the change in terms of a third weaker shock following the second of the pair. It seems, therefore, that the 60 to $100 \mathrm{msec}$. interval, in which facilitation of the response to a second stimulation occurs in the subject with myoclonus, may have some counterpart in normal cortical function, though no objective evidence of this has been found. 


\section{Discussion}

It is suggested that the potential changes that have been picked up from the scalp following stimulation of peripheral nerves are in fact cerebral action potentials and that they arise in the central or postcentral cerebral cortex. The short latency between the stimulus and the beginning of the response, of the order of 18 milliseconds following a stimulus at the elbow, suggests that the response indicates events occurring in the cortex at, or soon after, the time of arrival of the afferent volley at the cortex.

The failure to find a response to stimulation in two of the fourteen subjects examined was possibly due to the electrodes not being over the very limited area in which the response appears. That this was so is suggested by the fact that in one subject in whom no response was found in an earlier examination, a fuller investigation showed that a response could be detected. The same difficulty of locating the electrodes accurately over the centre of the disturbance may account for the difference in size of the deflections produced by the stimulation of the lateral popliteal nerve, Fig. 4C, and by stimulation of the median nerves, Fig. 4A and $\mathrm{B}$. It might be expected that the response to the stimulation of the median nerves would be the larger of the two. Although the primary positive wave is less clear during stimulation of the lateral poplitęal nerve than when stimulating the median nerve, the immediately succeeding negative change is considerably larger in the first instance. No significance may be attached to this difference since the electrode common to channels 1 and 2 on the right side, or to channels 3 and 4 on the left side, although well placed with respect to the active area in the coronal plane, may have been less favourably placed in the sagittal plane than was the midline electrode.

Comparison of the responses to stimulation of peripheral nerve described above with the potentials detected by Davis (1939) following auditory stimulation, and by Davis, Davis, Loomis, Harvey, and Hobart (1939) following electrical stimulation of a finger, is difficult on account of the differences in techniques used. The responses described by these authors had a latency of 30 to 40 msecs. and 40 to 100 msecs. respectively, and in both cases the duration of the response was described as of the order of $\mathbf{3 0 0}$ msecs. From these figures it seems' unlikely that the mechanisms involved are closely related.

The relation of these responses to peripheral nerve stimulation, consisting of a group of two to four waves, each with a period of 15 to 20 msecs., and a peak amplitude on the scalp of 5 to $10 \mu \mathrm{V}$., to the potential changes that may be evoked from the cortex of animals by sensory $\stackrel{\mathbb{D}}{=}$ stimulation is not clear on the evidence at present $\underline{O}$ available. The polarity of the first phase of the $Z$ wave in which the active area becomes positive $\stackrel{\circ}{=}$ with respect to other parts of the scalp, and its duration of 15 to 20 msecs., suggest that there may be some correspondence with the responses to 0 electrical stimulation of a toe in the monkey after $\stackrel{N}{\infty}$ recovery from ether anæsthesia that have been $\frac{\rho}{\mathcal{S}}$ described by Marshall, Woolsey, and Bard (1941). How the latencies of the responses correspond in the? two cases may not be considered in the absence of $\overrightarrow{\vec{F}}$ more information about the lengths of the pathways involved. It is interesting to note that in the record shown in Fig. 4A, though the primary wave is $\frac{\overline{\bar{N}}}{\bar{\rho}}$ visible only in traces 1 and 2 , the later negative $\frac{\pi}{\sigma}$ phase occurring 50 msecs. after the stimulus also $\stackrel{\mathbb{}}{\circ}$ appears in lead 3 . It might be suggested that कै this later discharge represents a less well localized $\vec{\circ}$ type of response similar to the secondary discharges described by Marshall and others. Before this may $\vec{\omega}$ be accepted evidence is needed, and is lacking in the present records, to show that the spread is not $\overline{3}$ a purely electrical effect, due to the size of the later ? discharge being greater than that of the primary $\overrightarrow{0}$ wave.

1. Electrical stimulation of peripheral nerves if fourteen healthy subjects has produced potential $\rightarrow$ changes on the scalp in twelve of them.

2. These potential changes occur over the contra lateral sensory area, near the midline when stimts lating the leg and more laterally when stimulating the arm.

3. The latency between the stimulus and the first peak of the response increases as the stimulus to the nerve is applied nearer to the periphery. It varies between 22 msecs. when stimulating at the elbows and 36 msecs. when stimulating at the knee.

4. Reasons are given for considering that these potential changes represent cerebral activity in the sensory area and are not artefacts.

\section{The significance of the findings is discussed.}

The author would like to express his indebtedness to the Engineer in Chief of the Post Office for the loan of the crystal oscillator used in these experiments, and to Mr. H. B. Law of the Post Office Research Station for advice in connexion with the frequency-dividing circuits used with this oscillator.

\section{REFERENCES}

Adrian, E. D., and Yamagiwa, K. (1935). Brain, 58, 323. Davis, P. A. (1939). J. Neurophysiol., 2, 494.

Davis, H., Davis, P. A.; Loomis, A. L., Harvey, E. N., and Hobart, G. (1939) $\mathrm{m}$ Ibid., 2, 500.

Marshall, W. H., Woolsey, C. N., and Bard, P. (1941). N Ibid., 4, 1. 